\title{
A NOTE ON AN ADAPTIVE ALGORITHM BASED ON CHEBYSHEV COEFFICIENTS FOR TWO-POINT BOUNDARY VALUE PROBLEMS
}

\author{
WOJCIECH L. GOLIK \\ University of Missouri, St. Louis - U.S.A.
}

\begin{abstract}
An adaptive version of an algorithm, first described by Greengard and Rokhlin, for numerical solution of two-point boundary value problems is proposed. The algorithm transforms two-point BVPs into integral equations, which are then solved by the Nyström method using Chebyshev quadratures. The dense system of algebraic equations is solved in recursively in $O(N)$ operations. The a posteriori node addition algorithm based on the size of Chebyshev coefficients of the solution approximations yields a robust method. The proposed approach combines the advantages of integral formulation and fast solution of dense linear systems with an automatic resolution of boundary and internal layers.
\end{abstract}

1991 Mathematics Subject Classification. Primary 65L10, 65L50; Secondary 65L12. 


\section{Two-point boundary value problems}

In a 1991 paper Greengard and Rokhlin [1] presented a numerical technique for a rapid solution of integral equations resulting from scalar linear twopoint boundary value problems (TPBVP) of the form

$$
u^{\prime \prime}(x)+p(x) u^{\prime}(x)+q(x) u(x)=g(x),
$$

for $x \in(c, d)$, where the functions $p, q, g$ are piecewise continuous, subject to the boundary conditions

$$
\begin{aligned}
& a_{c} u(c)+b_{c} u_{x}(c)=0, \\
& a_{d} u(d)+b_{d} u_{x}(d)=0 .
\end{aligned}
$$

Note, that non-homogeneous boundary conditions can be reduced to this form by an addition of an appropriate linear function to $g$. The method is based on an integral equation formulation of the TPBVP. Assuming that the Green function for the above problem is unavailable consider a related equation

$$
u^{\prime \prime}(x)+p_{0}(x) u^{\prime}(x)+q_{0}(x) u(x)=0 .
$$

with the functions $p_{0}, q_{0} \in C^{\mathrm{l}}(c, d)$ selected in such a way that the Green function, $G_{0}$ for equation (1.4) and boundary conditions (1.2),(1.3) (the background Green function) is explicitly known. It can be constructed from two independent solutions $u_{l}, u_{r}$ of (1.4), satisfying boundary conditions (1.2) and (1.3), respectively. Then the Green's function $G_{0}$ is given by

$$
G_{0}(x, y)=\left\{\begin{array}{lc}
u_{l}(x) v_{l}(y), & y<x \\
u_{r}(x) v_{r}(y), & x<y
\end{array}\right.
$$

where $v_{l}=u_{r} / s, v_{r}=u_{l} / s, s=u_{l}^{\prime} u_{r}-u_{r}^{\prime} u_{l}$. For example, if boundary conditions satisfy $\left|a_{c}\right|+\left|a_{d}\right| \neq 0$, selecting $p_{0}(x)=0, q_{0}(x)=0$ gives $u_{l}(x)=a_{c}(x-c)-b_{c}$ and $u_{r}(x)=a_{d}(x-d)-b_{d}$. For $\left|a_{c}\right|+\left|a_{d}\right|=0$ one can choose $p_{0}(x)=0, q_{0}(x)=-1$ with $u_{l}(x)=\cosh (x-c)$ and $u_{r}(x)=$ $\cosh (x-d)$.

The solution to the problem $(1.1),(1.2),(1.3)$ can be represented by the formula

$$
u(x)=\int_{c}^{d} G_{0}(x, y) \sigma(y) d y
$$


where $\sigma$ satisfies the integral equation

$$
\sigma(x)+\tilde{p}(x) \int_{c}^{d} G_{1}(x, y) \sigma(y) d y+\tilde{q}(x) \int_{c}^{d} G_{0}(x, y) \sigma(y) d y=g(x)
$$

and $\tilde{p}=p(x)-p_{0}(x), \tilde{q}=q(x)-q_{0}(x), G_{1}(x, y)=\frac{\partial}{\partial x} G_{0}(x, y)$.

In the next section we show how the solution $\sigma$ can be expressed as a linear combination of solutions of $3 N$ integral equations defined on $N$ subintervals of $[c, d]$. The integral equations on the subdomains are then solved numerically by the Nyström method [2] with Chebyshev quadratures [3]. in $O\left(N n^{3}\right)$ operations, where $n$ is the size of the quadrature. The TPBVP solution is obtained by a fast recursive merging of solutions of $3 N$ integral equations. The complete Greengard-Rokhlin algorithm solves a two-point boundary problem in $O(N)$ operations.

This paper describes an adaptive algorithm based on the size of Chebyshev quadrature coefficients. The key observation is that the size of these coefficients is related to the accuracy of solution over a given domain subinterval.

\section{Greengard-Rokhlin recursive algorithm}

A direct discretization of equation (1.7) gives rise to a dense system of $N$ linear algebraic equations resulting in an $O\left(N^{3}\right)$ algorithm, making the direct approach via integral equations expensive computationally. Paper [1] describes the algorithm which obtains numerical solution of equation (1.7) and rapid construction of $u$ from its representation (1.6) in $O(N)$ operations. It begins by reducing the problem to the solution of $3 N$ integral equations on subintervals of $(c, d)$ and then assembles them recursively to obtain $\sigma$ and ultimately $u$. Below we give a description of this recursive algorithm.

Following a standard convention we denote the restrictions of functions $f, g \in L^{2}(c, d)$ to $A \subset(c, d)$ by $f_{\mid A}, g_{\mid A}$ and their inner product by $(f, g)=\int_{c}^{d} f(x) g(x) d x$. Given an integer $m>0$, let $b_{1}, b_{2}, \ldots, b_{2^{m}+1}$ denote a strictly increasing sequence of points in $(c, d)$ with $b_{1}=c$ and $b_{2^{m}+1}=$ d. Define a hierarchy of $B_{i}^{m}$ intervals by setting $B_{i}^{m}=\left[b_{i}, b_{i+1}\right], \quad i=$ $1, \ldots, 2^{m}$ and $B_{i}^{k}=B_{2 i-1}^{k+1} \cup B_{2 i}^{k+1}, \quad i=0, \ldots m-1, \quad i=1, \ldots, 2^{k}$. Define a sequence of integral operators $P_{i}^{m}, P_{i}^{m}: L^{2}\left(B_{i}^{m}\right) \rightarrow L^{2}\left(B_{i}^{m}\right)$ by 


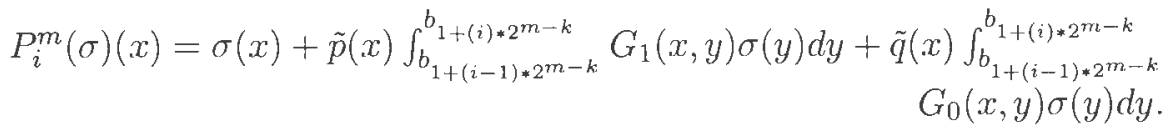

\section{Recursive algorithm}

Step 1. For each $i=1, \ldots, 2^{m}=M$ solve the following integral equations

$$
P_{i}^{m}\left(\eta_{i}^{m}\right)=g_{\mid B_{i}^{m}}, \quad P_{i}^{m}\left(\phi_{l_{i m}}\right)=\psi_{l_{\mid B_{i}^{m}}}, \quad P_{i}^{m}\left(\phi_{r_{i m}}\right)=\psi_{r_{\mid B_{i}^{m}}}
$$

where $\psi_{l}=\tilde{p} u_{l}^{\prime}+\tilde{q} u_{l}$ and $\psi_{r}=\tilde{p} u_{r}^{\prime}+\tilde{q} u_{r}$.

Step 2. For each $i=1, \ldots, 2^{m}$ compute the inner products

$$
\begin{array}{ll}
\alpha_{11}^{i, m}=\left(v_{l_{\mid B_{i}^{m}}}, \phi_{l_{i m}}\right), & \alpha_{12}^{i, m}=\left(v_{r_{\mid B_{i}^{m}}}, \phi_{l_{i m}}\right), \quad \delta_{l}^{i, m}=\left(v_{l_{\mid B_{i}^{m}}}, \eta_{i, m}\right), \\
\alpha_{21}^{i, m}=\left(v_{l_{\mid B_{i}^{m}}}, \phi_{r_{i m}}\right), & \alpha_{22}^{i, m}=\left(v_{r_{\mid B_{i}^{m}}}, \phi_{r_{i m}}\right), \quad \delta_{r}^{i, m}=\left(v_{r_{\mid B_{i}^{m}}}, \eta_{i, m}\right) .
\end{array}
$$

Step 3. Given $k=m-1, m-2, \ldots, 0$ compute recursively for each $i=$ $1, \ldots, 2^{k}$

$$
\begin{aligned}
\Delta^{i, k} & =1-\alpha_{12}^{2 i-1, k+1} \cdot \alpha_{21}^{2 i-1, k+1}, \\
\alpha_{11}^{i, k} & =\frac{\left(1-\alpha_{11}^{2 i, k+1}\right) \cdot\left(\alpha_{11}^{2 i-1, k+1}-\alpha_{12}^{2 i-1, k+1} \cdot \alpha_{21}^{2 i-1, k+1}\right)}{\Delta^{i, k}}+\alpha_{11}^{2 i-1, k+1}, \\
\alpha_{12}^{i, k} & =\frac{\alpha_{12}^{2 i-1, k+1} \cdot\left(1-\alpha_{22}^{2 i, k+1}\right) \cdot\left(1-\alpha_{11}^{2 i, k+1}\right)}{\Delta^{i, k}}+\alpha_{12}^{2 i, k+1}, \\
\alpha_{21}^{i, k} & =\frac{\alpha_{21}^{2 i, k+1} \cdot\left(1-\alpha_{22}^{2 i-1, k+1}\right) \cdot\left(1-\alpha_{11}^{2 i-1, k+1}\right)}{\Delta^{i, k}}+\alpha_{21}^{2 i-1, k+1}, \\
\alpha_{22}^{i, k} & =\frac{\alpha_{21}^{2 i, k+1} \cdot\left(1-\alpha_{22}^{2 i-1, k+1}\right) \cdot\left(1-\alpha_{11}^{2 i-1, k+1}\right)}{\Delta^{i, k}}+\alpha_{21}^{2 i-1, k+1}, \\
\delta_{l}^{i, k} & =\frac{\left(1-\alpha_{11}^{2 i, k+1}\right)}{\Delta^{i, k}} \cdot \delta_{l}^{2 i-1, k+1}+\delta_{l}^{2 i, k+1}-\frac{\left(1-\alpha_{11}^{2 i, k+1}\right) \cdot \alpha_{12}^{2 i-1, k+1}}{\Delta^{i, k}} \cdot \delta_{r}^{2 i, k+1}, \\
\delta_{r}^{i, k} & =\frac{\left(1-\alpha_{22}^{2 i-1, k+1}\right)}{\Delta^{i, k}} \cdot \delta_{r}^{2 i, k+1}+\delta_{r}^{2 i-1, k+1}-\frac{\left(1-\alpha_{22}^{2 i-1, k+1}\right) \cdot \alpha_{21}^{2 i, k+1}}{\Delta^{i, k}} \cdot \delta_{l}^{2 i, k+1} .
\end{aligned}
$$

Step 4. Let $\lambda_{l}^{1,0}=\lambda_{r}^{1,0}=0$. For $k=0, \ldots, m-1$ compute recursively for each $i=1, \ldots, 2^{k}$

$$
\lambda_{l}^{2 i-1, k+1}=\lambda_{l}^{i, k},
$$




$$
\begin{aligned}
\lambda_{r}^{2 i-1, k+1} & =-\frac{\left(1-\alpha_{11}^{2 i-1, k+1}\right) \cdot \alpha_{21}^{2 i, k+1}}{\Delta^{i, k}} \cdot \lambda_{l}^{i, k}+\frac{\left(1-\alpha_{22}^{2 i, k+1}\right)}{\Delta^{i, k}} \cdot \lambda_{r}^{i, k} \\
& +\frac{\alpha_{21}^{2 i, k+1} \cdot \delta_{l}^{2 i-1, k+1}-\delta_{r}^{2 i, k+1}}{\Delta^{i, k}}, \\
\lambda_{l}^{2 i, k+1} & =-\frac{1-\alpha_{11}^{2 i-1, k+1}}{\Delta^{i, k}} \cdot \lambda_{l}^{i, k}+\frac{\left(1-\alpha_{22}^{2 i, k+1}\right) \cdot \alpha_{12}^{2 i-1, k+1}}{\Delta^{i, k}} \cdot \lambda_{r}^{i, k} \\
& +\frac{\alpha_{12}^{2 i-1, k+1} \cdot \delta_{r}^{2 i, k+1}-\delta_{l}^{2 i-1, k+1}}{\Delta^{i, k}}, \\
\lambda_{r}^{2 i, k+1} & =\lambda_{r}^{i, k} .
\end{aligned}
$$

Step 5. For each $i=1, \ldots, 2^{m}$ compute the solution $\sigma$ of equation (1.7) for $x \in B_{i}^{m}$ from

$$
\sigma(x)=\eta_{i}^{m}(x)+\lambda_{l}^{i, m} \cdot \phi_{l_{i m}}(x)+\lambda_{r}^{i, m} \cdot \phi_{r_{i m}}(x)
$$

Step 6. Given $\sigma$ compute the solution $u$ from equation (1.6) in $O\left(2^{m}\right)$ operations.

The numerical implementation of this algorithm is made via the application of Nyström method with Chebyshev quadratures (see [1] for details). This results in replacing Step 1 with

Step $\tilde{1}$. For each $i=1, \ldots, 2^{m}$ construct and solve via Gauss elimination three $n \times n$ linear systems

$$
A_{\mid B_{i}^{m}}^{n}\left(\tilde{\eta}_{i}^{m}\right)=\tilde{f}_{\mid B_{i}^{m}}, \quad A_{\mid B_{i}^{m}}^{n}\left(\tilde{\phi}_{l_{i m}}\right)=\tilde{\psi}_{l_{\mid B_{i}^{m}}}, \quad A_{\mid B_{i}^{m}}^{n}\left(\tilde{\phi}_{r_{i m}}\right)=\tilde{\psi}_{r_{B_{i}^{m}}^{m}},
$$

The matrices $A_{\mid B_{i}^{m}}^{n}$ are defined by

$(2.2) A_{[a, b]}^{n}=I+\tilde{P}\left(U_{l}^{\prime} S_{[a, b]}^{n} V_{l}+U_{r}^{\prime} \tilde{S}_{[a, b]}^{n} V_{r}\right)+\tilde{Q}\left(U_{l} S_{[a, b]}^{n} V_{l}+U_{r} \tilde{S}_{[a, b]}^{n} V_{r}\right)$,

where $\tilde{P}, \tilde{Q}, U_{l}^{\prime}, U_{l}, U_{r}^{\prime}, U_{r}, V_{l}, V_{r}: R^{n} \rightarrow R^{n}$ are diagonal matrices and $S_{[a, b]}^{n}$ and $\tilde{S}_{[a, b]}^{n}$ represent the left and right spectral integration operators (describing the Chebyshev quadrature) [1].

Step 2 and Step 6 also require an application of $n$-point Chebyshev quadratures to approximate the inner products. The operation count of the algorithm is as follows. The solution of each integral equation requires 
$O\left(n^{3}\right)$ operations which amounts to no more than $O\left(M n^{3}\right)$ operations for Step 1. The inner products computed in Step 2, each approximated by n-order Chebyshev quadrature, require $O\left(n^{2}\right)$ operations for the total of $O\left(M n^{2}\right)$ flops. The recursive steps 3 and 4 require $O(M)$ operations, step 5 requires $O(M n)$ flops, and recursive computations in step 6 are of order $O(M n)$. The total work is of order $O\left(M n^{3}\right)$, and since the total number of evaluation points (nodes) is $N=M n$, the CPU estimate of GreengardRokhlin algorithm is $O\left(N n^{2}\right)$, i.e. it is proportional to the number of nodes $N$.

\section{Adaptive algorithm}

As mentioned in section 2, the Greengard-Rokhlin algorithm is not limited to the recursive bisection of the original interval $[c, d]$ into $2^{m}$ subintervals. It is possible to conceive an adaptive version of the recursive algorithm, very closely related to standard adaptive quadratures. The main step of such an algorithm starts with an interval, approximates the solution on it (via $n$-point Chebyshev quadrature), estimates the error, and decides if a further interval bisection is needed. In case of the affirmative answer, the refinement step is repeated recursively on each of the subintervals until no more bisections are necessary, or the total number of subintervals cxcceds some predetermined level.

To estimate the solution error over a given subinterval consider two model equations

$$
f(x)+\int_{a}^{b} K(x, y) f(y) d y=g(x)
$$

and

$$
A_{[a, b]}^{n} \tilde{f}^{n}=g^{n} .
$$

Recail that vector $\tilde{f}^{n}$ in equation (3.2) represents an approximation to values of $f$ at Chebyshev nodes $\tilde{f}^{n} \approx\left(f\left(\tau_{1}\right), \ldots, f\left(\tau_{n}\right)\right)$. To approximate function $f$ over the whole interval $[a, b]$ we define

$$
\tilde{f}(x)=\sum_{j=0}^{n-1} \alpha_{j} T_{j}\left(\frac{2(x-a)}{b-a}-1\right),
$$

where coefficients $\alpha_{j}$ are obtained from $\tilde{f}^{n}$ via the $n$-point discrete cosine transform $\mathcal{C}_{n}$. To analyze the error $\|f-\tilde{f}\|$ in some function norm, we define 
two auxiliary operators. Let $B_{n}$ denote the evaluation operator which maps any continuous function $f$ on interval $[a, b]$ to the set $\left(f\left(\tau_{1}\right), \ldots, f\left(\tau_{n}\right)\right)$ of values of $f$ at the Chebyshev nodes in $[a, b]$. Let $C_{n}$ denote the operator mapping $n$-element vector $\tilde{h}^{n}=\left(h_{1}, \ldots, h_{n}\right)$ to the $n$-term Chebyshev expansion $\tilde{h}(x)=\sum_{j=0}^{n-1} a_{j} T_{j}\left(\frac{2(x-a)}{b-a}-1\right)$ such that $\mathcal{C}_{n} \tilde{h}^{n}=\left(a_{0}, \ldots, a_{n-1}\right)$. Note that the operators satisfy

$$
B_{n} C_{n} \tilde{h}^{n}=\tilde{h}^{n},
$$

for any $n$-element vector $\tilde{h}^{n}$. Observe that it is now possible to modify equation (3.2) to

$$
C_{n} A_{[a, b]}^{n} B_{n} C_{n} \tilde{f}^{n}=C_{n} g^{n},
$$

which can be written in the form

$$
\tilde{A} \tilde{f}(x)=\tilde{g}(x),
$$

where $\tilde{A}=C_{n} A_{[a, b]}^{n} B_{n}, \tilde{g}=C_{n} g^{n}$, and $\tilde{f}$ is defined in (3.3). The relative error between $\tilde{f}(x)$ and $f(x)$ is given by [2]

Theorem 1. Rewrite the integral equation (3.1) in the operator notation as $A f=g$ and assume that operator $A$ is invertible. Then

$$
\frac{\|f-\tilde{f}\|}{\|f\|} \leq \kappa(A)\left(\frac{\|(A-\tilde{A}) \tilde{f}\|}{\|f\|}+\frac{\|g-\tilde{g}\|}{\|f\|}\right),
$$

where $\kappa(A)$ is the condition number of operator $A$ relative to the function norm $\|\cdot\|$.

The above theorem indicates that the relative error of the $n$-point Chebyshev approximation to the solution of equation (3.1) depends on $\kappa(A)$ as well as the size of $\frac{\|g-\tilde{g}\|}{\|f\|}$ and $\frac{\|(A-\tilde{A}) \tilde{f}\|}{\|f\|}$. The size of $\kappa(A)$ is given by the problem and can not be controlled. The term $\frac{\|g-\tilde{g}\|}{\|f\|}$ can be made small by assuring that $[a, b]$ is small enough, so that $g$ can be accurately resolved by $n$ Chebyshev coefficients. The size of the remaining term can be controlled by estimating $\|(A-\tilde{A}) \tilde{f}\|$.

Recalling equation 2.2 we observe that $\|(A-\tilde{A}) \tilde{f}\|=0$. as long as $\tilde{f}$ is a polynomial of degree $n-2$ and $v_{l}, v_{r}$ are polynomials of degree 1 (see the remark following equation (1.5)). This is the consequence of the exactness of $n$-point Chebyshev quadratures for $n-1$ polynomials and the definition of $\tilde{A}$. For $\tilde{f}$ to be a polynomial of degree $n-2$, one must have 
$\alpha_{n-2}=\alpha_{n-1}=0$ in equation (3.3). If, on the other hand, the last two Chebyshev coefficients for $\tilde{f}$ are merely small,

$$
\left\|\alpha_{n-2}\right\|<\epsilon, \quad\left\|\alpha_{n-1}\right\|<\epsilon, \quad 0<\epsilon<<1,
$$

it follows that

$$
A \tilde{f}-\tilde{A} \tilde{f}=O(\epsilon) .
$$

This observation is a basis of the following refinement strategy used to produce a better mesh.

\section{Refinement Algorithm}

- At each refinement level $m$ determine Chebyshev coefficients $\alpha_{j}$ of the solution $u$ of equation (1.6) on each subinterval $B_{i}^{m}$ using the recursive algorithm from section 2 .

- For each $B_{i}^{m}$ compute the monitor $M_{i}=\max \alpha_{n-2}, \alpha_{n-1}$

- subdivide $B_{i}^{m}$ if $M_{i}<\epsilon$, for some, user defined tolerance TOL.

The adaptive algorithm starts with just one interval $[c, d]$ and is applied recursively. Each bisection of the parent interval requires the solution of three linear systems (see step $\tilde{1}$ ) on each of the two children intervals. Once these are obtained they are merged with the solution on other intervals which are stored from the previous step. Thus if only $p$ intervals are bisected at a given level of refinement, the CPU cost is only $O\left(p n^{3}\right)$ for the linear system step and $O(M n)$ for the merging step, where $M$ is the number of intervals at a given refinement level. This complexity of the algorithm suggests that it is unnecessary to nerge any of the intervals (in case the corresponding Chebyshev coefficients are extremely small).

Remark. Successively refining the mesh will eventually result in adequately resolved approximate solutions i.e. the high order Chebyshev coefficients of their restrictions to intervals $B_{i}$ (dilated back to $[-1,1]$ ) being negligible. The algorithm will always terminate. Once the algorithm stops refining the mesh, it is important to ascertain the accuracy of the solution. Letting $u_{n}$ denote the approximation to the solution on the refined grid, obtained with the help of $n$-point Chebyshev quadratures, we decided to recompute the approximation on the same grid with the help $\tilde{n}$-point Chebyshev quadratures, where $\tilde{n}=n+2$. Denoting that approximation by $u_{\tilde{n}}$ we estimate the error in $u_{n}$ as

$$
\left\|u-u_{n}\right\| \approx\left\|u_{\tilde{n}}-u_{n}\right\| .
$$




\section{Numerical Examples}

This section presents results of some numerical tests for problems with boundary layers, turning points, and spikes. The examples illustrate selfadaptive features of the proposed algorithm.

\section{Example 1 :}

Consider the Dirichlet problem on interval $[0,1] \epsilon u^{\prime \prime}+u=0$, where $u(x)=$ $\exp \left(-x / \epsilon^{1 / 2}\right)$. For $0<\epsilon<<1$ the problem exhibits a boundary layer at $x=0$. We set $\epsilon=1 E-6$ and $T O L=1 E-6$ using the adaptive algorithm with $n=8$. The mesh sequence is

$$
1[1], 2[1], 3[1], 4[1], 5[1], 6[2], 7[3], 8[4], 9[5], 10[6], 12[8], 14[10]
$$

where the number of mesh points in the interval $[0,0.05]$ is given in brackets. The actual error for the last mesh is $.38 E-8$ and the error estimate $.6 E-$ 7. Similar accuracy using the Greengard-Rokhlin algorithm without mesh refinement is not achieved until $M=1000$.

\section{Example 2 :}

Consider the Dirichlet problem $\epsilon u^{\prime \prime}+x u^{\prime}=f$ on interval $[-1,1]$ with the solution $u(x)=\cos (\pi x)+\operatorname{erf}\left(x /(2 \epsilon)^{1 / 2}\right) / \operatorname{er} f\left(1 /(2 \epsilon)^{1 / 2}\right)$. For $0<\epsilon \leq 1$ the solution has an internal layer (turning point) at $\mathrm{x}=0$ (see Fig. ??). We solve this problem with $\epsilon=1 E-4$ and $T O L=1 E-6$ using the adaptive algorithm with $n=8$. The mesh sequence is

$$
1[1], 2[1], 4[1], 8[1], 16[1], 32[1], 36[3], 40[7], 44[11], 48[15] .
$$

The actual error for the last mesh is $.18 E-8$ and the error estimate. $1 E-7$. This BVP problem was adapted from [4], where the adaptive BVP solver COLSYS used 132 subintervals to obtain the solution with the given tolerance. 

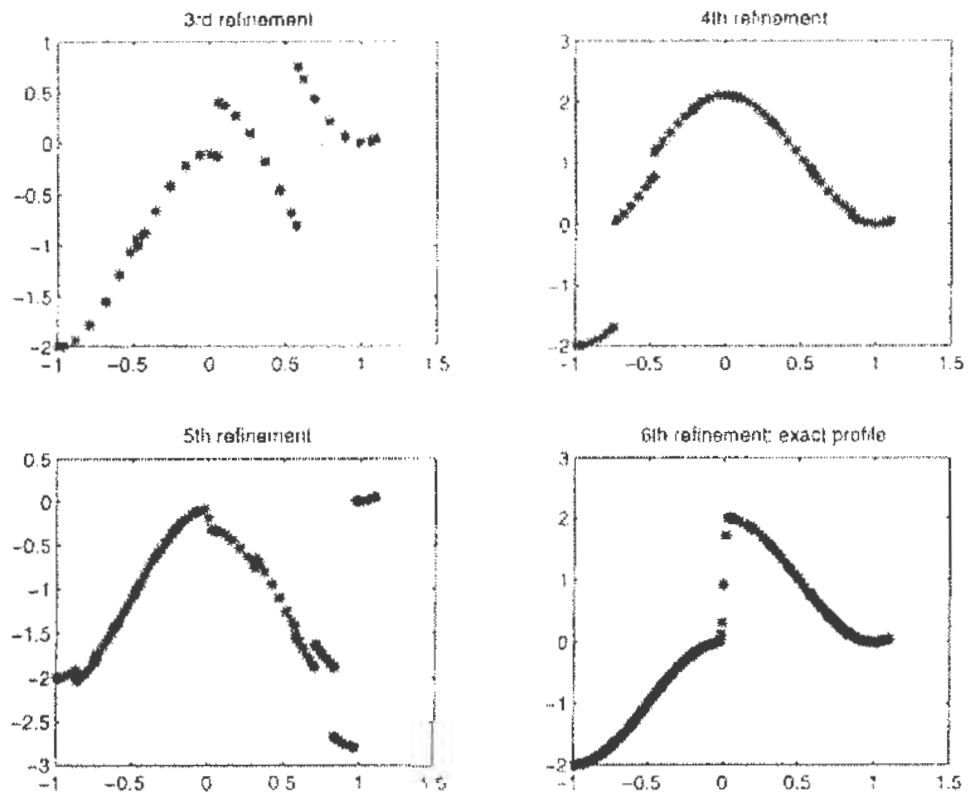

Figure 1 : Example 2: an internal layer. Solution approximations at different refinements with $\mathrm{M}=4,8,16,32$. 


\section{Example 3 :}

Consider the Dirichlet problem

$$
u^{\prime \prime}+\frac{2 \epsilon-6(0.24-x)^{2}}{\left(\epsilon+(0.24-x)^{2}\right)^{2}} u=0
$$

on the interval $[0,1]$ with the solution $u(x)=\frac{1}{\left.\epsilon+(0.24-x)^{2}\right)^{2}}$ exhibiting a peak at $x=0.24$ for small $\epsilon$. The problem with $\epsilon=1 E-4$ is solved for $T O L=1 E-6$ using the adaptive algorithm with $n=8$. The mesh sequence is

$$
1,2,4,6,8,10,13,25,20,41,44 \text {. }
$$

The actual error for the last mesh is $.8 E-3$. This BVP problem was copied from [5], where the adaptive BVP solver COLSYS needed 80 subintervals to obtain the solution for $\epsilon=5 E-3$ within the same tolerance.

\section{Example 4:}

Consider the Dirichlet problem $\epsilon u^{\prime \prime}+u=f$ on $[0,1]$, where $u(x)=\exp \left(-x / \epsilon^{1 / 2}\right)+$ $\exp \left((1-x) / \epsilon^{1 / 2}\right)+\sin 2 \pi x$. For $0<\epsilon<<1$ the solution exhibits boundary layers in addition to variation due to $\sin 2 \pi x$. Notice that the boundary values at both ends are equal to 1 We set $\epsilon=1 E-6$ and $T O L=1 E-6$ and solve the problem using the adaptive algorithm with $n=8$. The mesh sequence is

$$
1,2,4,8,16,32,46,52,54,58,60 \text {. }
$$

The actual error for the last mesh is $.24 E-6$ and the error estimate $.6 E-6$. 

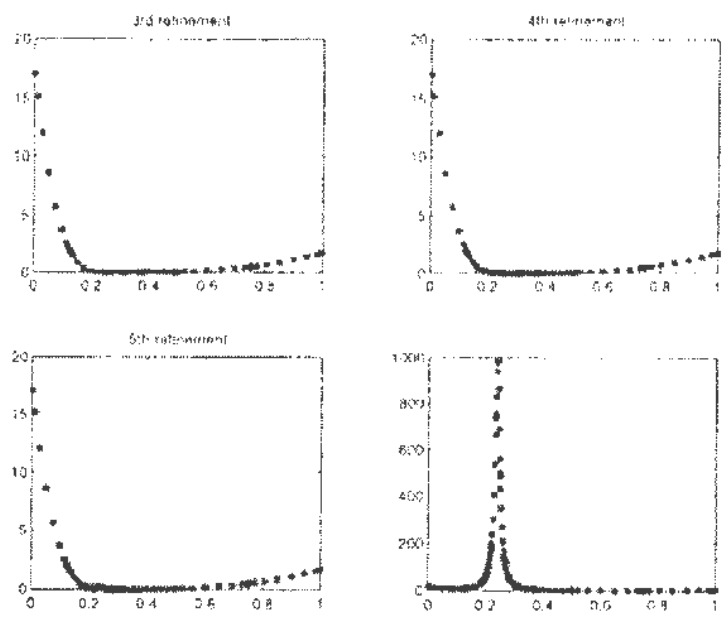

Figure 2 : Exaunple 3: a spike. Solntion approximations at different refinements with $M=6,8,10,13$. First appearance of the spike occurs when $M=13$.
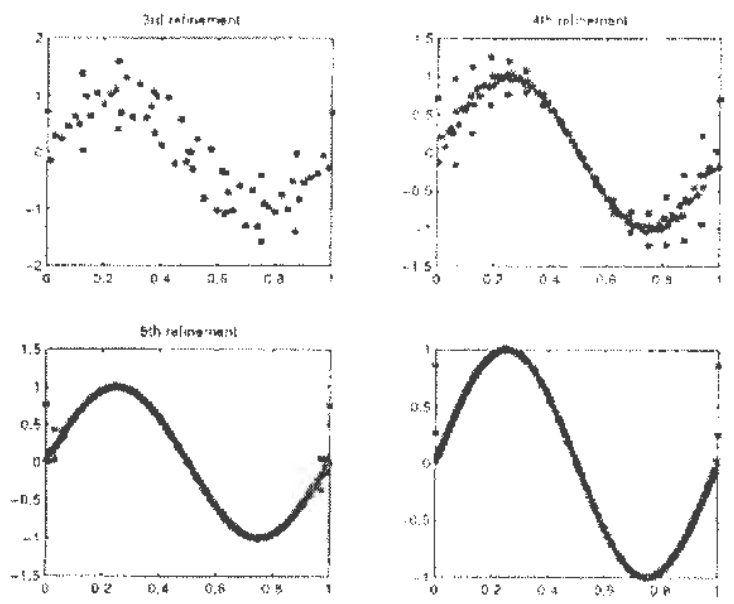

Figure 3: Example 4: boundary layers. Solution approxinations at different refinements with $\mathrm{M}=6,8,13,25$. 


\section{Acknowledgment}

The author would like to thank Professors Rokhlin and Greengard for the use of their original code.

\section{References}

[1] L. Greengard and V. Rokhlin. On the Numerical Solution of TwoPoint Boundary Valuc Problems. Communications on Pure and Applied Mathematics, 44 : pp. 419-452, (1991).

[2] C. T. H. Baker. The Numerical Treatment of Integral Equations. Clarendon Press, Oxford, (1977).

[3] C. W. Clenshaw and A. R. Curtis. A method for numerical integration on an automatic computer. Numer. Math., 2 : pp. 197-205, (1960).

[4] U. M. Ascher, R. M. M. Matheij, and R. D. Russell. Numerical solution of boundary value problems for ordinary dfferential equations. Series in Computational Mathematics. Prentice Hall, (1988).

[5] J. R. Cash. On the numerical integration of nonlinear two point boundary value problems using iterated deferred corrections, Part II. SIAM J. Numer. Anal., 25 : pp. 877-898, (1988).

Received : September 1998.

\section{W. L. Golik}

Department of Mathematics and Computer Science

University of Missouri - St. Louis

St. Louis, Missouri 63121

U. S. A. 